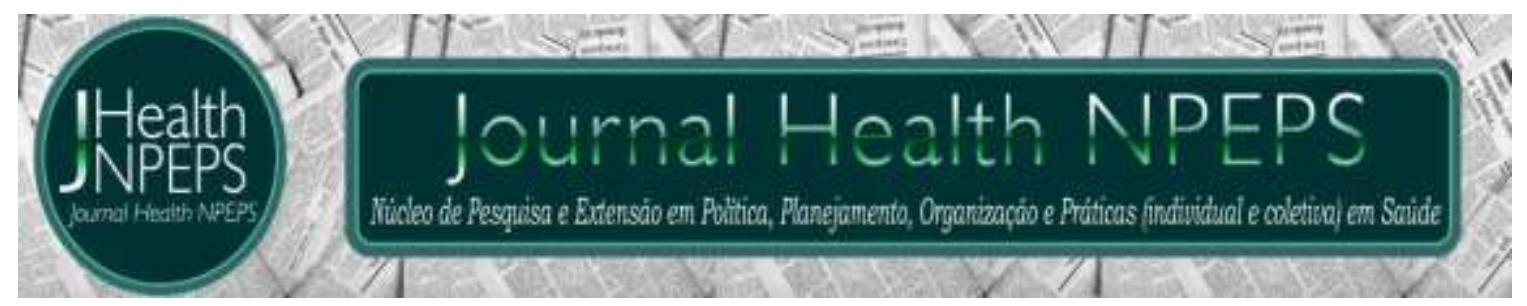

http://dx.doi.org/10.30681/252610104365

ARTIGO ORIGINAL

\title{
Conhecimento de enfermeiros sobre sepse e choque séptico em um hospital escola
}

\section{Nurses' knowledge about sepsis and septic shock in a teaching hospital \\ Conocimiento de las enfermeras sobre sepsis y shock séptico en un hospital universitario}

\section{Thais Vilela de Sousa ${ }^{1}$, Lorena Morena Rosa Melchior², Maria Luiza Rêgo Bezerra $^{3}$, Francidalma Soares Souza Carvalho Filha ${ }^{4}$, Osmar Pereira dos Santos $^{5}$, Mayara Cândida Pereira ${ }^{6}$, Keila Cristina Félis ${ }^{7}$, lel Marciano de Moraes Filho ${ }^{8}$}

RESUMO

\begin{abstract}
${ }^{1}$ Enfermeira. Mestre e Doutoranda em Enfermagem pelo Programa de Pós-Graduação em Enfermagem da Universidade Federal de Goiás (UFG). Goiânia, Goiás, Brasil. E-mail: thais.fen@hotmail.com ORCID ID: https://orcid.org/0000-0002-7498-516X Autor principal - Endereço para correspondência: Rua 227, N 320 - Setor Leste Universitário, Goiânia - GO, CEP 74605-080.

${ }^{2}$ Enfermeira. Mestre e Doutoranda em Enfermagem pelo Programa de Pós-Graduação em Enfermagem da Universidade Federal de Goiás (UFG). Goiânia, Goiás, Brasil. E-mail: lorena_melchior@hotmail.com ORCID ID: https://orcid.org/0000-0002-8644-1784

${ }^{3}$ Enfermeira. Mestre em Enfermagem. Doutoranda em Bioética. Docente do curso de Enfermagem da Universidade Paulista (UNIP). Brasília, DF, Brasil. E-mail: marialuizaregobezerra@gmail.com ORCID ID: https://orcid.org/0000-0002-3336-7760

${ }^{4}$ Enfermeira. Doutora em Saúde. Docente da Universidade Estadual do Maranhão (UEMA). Balsas, Maranhão, Brasil. E-mail: francidalmafilha@gmail.com ORCID ID: https://orcid.org/0000-0001-5197$\underline{4671}$

${ }^{5}$ Enfermeiro. Mestre em Ciências Ambientais. Doutorando em Educação. Docente do curso de Enfermagem da Faculdade União de Goyazes Trindade. Trindade, Goiás, Brasil E-mail: osmarenfi@gmail.com ORCID ID: https://orcid.org/0000-0002-7962-622X

${ }^{6}$ Enfermeira. Mestre e Doutoranda em Gerontologia da Universidade Católica de Brasília. Coordenadora do curso de Enfermagem da Universidade Paulista (UNIP). Brasília, DF, Brasil. E-mail: enfamayara@gmail.com ORCID ID: https://orcid.org/0000-0002-0242-6262

${ }^{7}$ Enfermeira. Mestre em Ciências Ambientais e saúde. Docente do curso de Enfermagem do Centro Universitário de Goiatuba. Goiatuba, Goiás, Brasil. E-mail: keilafelis@hotmail.com ORCID ID: https://orcid.org/0000-0002-2532-6010

${ }^{8}$ Enfermeiro. Mestre em Ciências Ambientais e Saúde. Doutorando em Sociedade, Tecnologia e Meio Ambiente. Docente do curso de Enfermagem da Universidade Paulista (UNIP). Brasília, DF, Brasil. E-mail: ielfilho@yahoo.com.br ORCID ID: https://orcid.org/0000-0002-0798-3949
\end{abstract}

Este artigo está licenciado sob forma de uma licença Creative Commons Atribuição 4.0 Internacional, que permite uso irrestrito, distribuição e reprodução em qualquer meio, desde que a publicação original seja corretamente citada. 
Objetivo: identificar o conhecimento de enfermeiros sobre sepse e choque séptico em um hospital escola. Método: estudo transversal e descritivo, realizado em hospital escola público de uma capital brasileira. A amostra foi composta por 47 enfermeiros, que realizavam cuidados a pacientes adultos em unidades de internação. A coleta de dados ocorreu entre julho e agosto de 2017. Os resultados foram apresentados por estatística descritiva. Resultados: a maioria dos enfermeiros estudaram sobre sepse durante a graduação, mas $40,4 \%$ referiram ter dificuldades no cuidado ao paciente séptico, $70,2 \%$ responderam erroneamente sobre a atual definição de sepse, $51 \%$ ou elencaram sinais não utilizados para identificação da sepse ou descreveram apenas sinais da síndrome da resposta inflamatória sistêmica. Conclusão: é possível refletir que talvez esses participantes apenas "fazem" ou seguem prescrições. Esses participantes apresentaram pouco conhecimento acerca da definição e classificação da sepse, bem como dificuldades na identificação precoce das alterações sistêmicas.

Descritores: Sepse; Enfermagem; Conhecimento.

\section{ABSTRACT}

Objective: to identify nurses' knowledge about sepsis and septic shock in a teaching hospital. Method: cross-sectional descriptive study carried out in a public hospital in a brazilian capital. The sample consisted of 47 nurses who provided care to adult patients in inpatient units. Data collection took place between July and August 2017. The results were presented using descriptive statistics. Results: most nurses studied about sepsis during graduation, but 40.4\% reported having difficulties in the care of septic patients, $70.2 \%$ answered incorrectly about the current definition of sepsis and 51\% either listed unused signs to identify sepsis or described only signs of the systemic inflammatory response syndrome. Conclusion: it is possible to reflect that perhaps these participants only "do" or follow prescriptions. These participants had little knowledge about the definition and classification of sepsis, as well as difficulties in the early identification of systemic changes.

Descriptors: Sepsis; Nursing; Knowledge.

\section{RESUMEN}

Objetivo: identificar el conocimiento de las enfermeras sobre sepsis y shock séptico en un hospital universitario. Método: estudio descriptivo transversal realizado en hospital público de la capital brasileña. La muestra consistió en 47 enfermeras que brindan atención a pacientes adultos en unidades de hospitalización. Resultados: la mayoría de las enfermeras estudiaron sobre la sepsis durante el grado de Enfermería, pero $40.4 \%$ informó dificultades para tratar a los pacientes sépticos y el $70.2 \%$ respondió equivocadamente acerca de la actual definición de sepsis. Además, 51\% eligieron señales inusuales de identificación, o describieron solo los signos del síndrome de respuesta inflamatoria sistémica. Conclusión: es posible reflejar que quizás estos participantes simplemente "hacen" o siguen las prescripciones. Estos participantes tenían poco conocimiento sobre la definición y clasificación de la sepsis, así como dificultades en la identificación temprana de cambios sistémicos.

Descriptores: Sepsis; Enfermería; Conocimiento. 


\section{INTRODUÇÃO}

A sepse é uma síndrome complexa causada pela resposta inflamatória sistêmica descontrolada do indivíduo, originada por foco infeccioso, caracterizada por múltiplas manifestações clínicas que podem determinar disfunção orgânica ou morte. De acordo com a classificação atual do agravo, "sepse está definida como disfunção orgânica potencialmente fatal causada por uma resposta do hospedeiro desregulada à infecção" e choque séptico como "um subconjunto da sepse em que a circulação e as anormalidades celulares e metabólicas são profundas 0 suficiente para aumentar substancialmente a mortalidade"1.

Segundo o Instituto Latino Americano de Sepse, aproximadamente 15 a 17 milhões de pessoas no mundo são acometidas por esse agravo em um ano, sendo que 5 milhões evoluem para óbito². No Brasil, em 2018, foram registrados 19.604 óbitos por sepse $^{3}$. E apesar dos diversos avanços e estudos sobre os mecanismos fisiopatológicos da doença e o desenvolvimento de estratégias de manejo e intervenção ${ }^{1}$ as taxas de mortalidade ainda permanecem altas ${ }^{2}$.

A mortalidade na sepse está associado não somente às condições próprias do paciente, mas também ao início da instituição do tratamento. As recomendações atuais direcionam para instituição da terapêutica no primeiro atendimento, muito antes da internação em UTI. Entretanto, a promoção de um tratamento de início precoce requer 0 reconhecimento igualmente precoce do quadro. Deve-se entender o tempo como fator de extrema relevância, pois o menor tempo de reconhecimento é associado a um melhor prognóstico ${ }^{4}$.

Este reconhecimento ainda pode ser dificultado pela inespecificidade dos sinais $e$ sintomas, como hipotensão, hipóxia, redução do débito urinário ou mesmo sinais de resposta inflamatória como aumento das frequências cardíaca, respiratória e febre. Constante reavaliação das 
respostas humanas é de responsabilidade da enfermagem, especialmente do enfermeiro, num processo contínuo e ininterrupto de cuidado, e regido pelos preceitos da sistematização e do processo de enfermagem ${ }^{5}$.

0 conhecimento dos profissionais para a realização da identificação precoce pode evitar um mau prognóstico, os altos custos de assistência à saúde a esses pacientes e os riscos de comorbidades relacionadas às disfunções orgânicas. Esses fatores estão diretamente relacionados ao risco de morte e faz-se necessário conhecimento dos profissionais enfermeiros para intervir com eficácia, acurácia e qualidade uma vez que este são os profissionais mais próximos do paciente durante o período de internação $0^{5,6}$.

Nesse contexto, a equipe de enfermagem tem papel fundamental na avaliação clínica precoce por participar de forma direta no atendimento ao paciente com sepse e/ou choque séptico desde o primeiro momento. De acordo com Instituto LatinoAmericano de Sepse, em 2016, cerca de 2.552 casos de 32.694 de sepse e choque séptico foram tratados em enfermarias ou unidades de internação com mortalidade de 47,7\% .

Nessa perspectiva, é fundamental que a equipe de enfermagem dos setores de internação e pronto socorro, sobretudo, o enfermeiro, tenha conhecimento para reconhecer a sepse, identificar precocemente e direcionar o tratamento adequado considerando sua presença, quase que totalitária, beira leito no cuidado aos pacientes assistidos, caracterizando assim, sua imprescindível importância ${ }^{5,8}$.

A partir do exposto, surge 0 questionamento: Qual nível de conhecimento sobre sepse dos enfermeiros que atuam em unidades de internação e pronto socorro? Destarte, este estudo justifica-se pela alta incidência e mortalidade pela sepse e, primordialmente, pela importância da identificação e reconhecimento precoce desse agravo por profissionais da área da saúde, assim como por enfermeiros, visando oferecer uma assistência segura e de qualidade. 0 objetivo deste estudo é identificar o 
conhecimento de enfermeiros sobre sepse e choque séptico em um hospital escola.

\section{MÉTODO}

Trata-se de um estudo transversal e descritivo, realizado em um hospital escola público de uma capital brasileira. 0 período de coleta de dados foi de julho a agosto de 2017. A população de 63 enfermeiros realizava cuidados a pacientes adultos nas unidades de internação sendo esta então considerada para o estudo. As Unidades de Terapia Intensiva foram excluídas uma vez que o paciente séptico que lá se encontra, muitas vezes já está em franca disfunção orgânica e/ou em choque séptico ${ }^{9}$ não se tratando mais do reconhecimento precoce do agravo. Também foram excluídos setores pediátricos, uma vez que os sinais de sepse em crianças são ainda mais inespecíficos que no adulto ${ }^{10}$.

Foi critério de inclusão ser enfermeiro atuante no cuidado direto a pacientes adultos internados e de exclusão enfermeiros que estivessem de férias, licença médica ou licença maternidade. Assim, não contabilizando também as recusas, a amostra não-probabilística do tipo conveniência foi composta por 47 enfermeiros.

Os enfermeiros foram abordados durante seu plantão ou turno de trabalho, foram convidados a participar da pesquisa, foram esclarecidos quanto aos objetivos, e então a coleta de dados foi iniciada, posterior a assinatura do Termo de Consentimento Livre e Esclarecido (TCLE).

0 instrumento de coleta de dados foi criado pela própria equipe de pesquisa, baseado nas atuais recomendações ${ }^{1,4,5}$ e foi avaliado por um expert no assunto. Assim, os indivíduos responderam ao questionário semiestruturado contendo: (i) variáveis sociodemográficas (sexo, idade, tempo de trabalho na instituição, tempo de graduação e vínculo empregatício); (ii) questões específicas sobre o conhecimento adquirido e experiência no cuidar de paciente em sepse (questões direcionadas sobre definição, classificação da sepse, 
manifestações clinicas, tratamentos e bundles/recomendações).

A coleta de dados ocorreu individualmente em ambiente reservado escolhido pelo próprio enfermeiro e dentro da própria unidade de internação. Foi então Ihe oferecido o questionário para autopreenchimento e lhe concedido todo o tempo que o mesmo julgou necessário, de forma que o pesquisador aguardou 0 preenchimento completo pelo enfermeiro participante.

Os dados foram digitados e organizados em planilhas do excel e analisados usando o programa Statistical Package for Social Science (SPSS). As variáveis foram agrupadas por similaridade de conteúdo e os resultados apresentados por meio de estatística descritiva simples expressos por frequências absolutas (n) e frequências relativas (\%).

Todos os preceitos éticos e de responsabilidade em pesquisa foram respeitados conforme a Resolução $n^{\circ}$ 466/2012 e o projeto foi aprovado pelo Comitê de Ética em Pesquisa Humana do Hospital das Clínicas da Universidade Federal de Goiás por meio do parecer $\mathrm{n}^{\circ} 2.098989$ e CAAE 68007217.0.0000.5078.

\section{RESULTADOS E DISCUSSÃO}

A amostra estudada era em sua maioria do sexo feminino (93,6\%, $n=44)$, com faixa etária entre 23 e 60 anos, idade média de 38,5 anos. Em sua maior fração, casada $(48,9 \% ; n=23)$, há mais de 10 anos atuando na instituição $(40,4 \% ; n=19)$ e com mais de um vínculo empregatício $\quad(38,29 \%$; $n=18)$.

Em relação a sua formação, a mediana do tempo na instituição era 14,5 anos (1- 28 anos) e do tempo de graduação 14 anos (1-36 anos). Dentre os participantes, $66 \%$ $(n=31)$ eram especialistas em saúde mental, terapia intensiva, saúde pública e da família e saúde do trabalho; $6,4 \% \quad(\mathrm{n}=3)$ graduados; $25,5 \%(n=12)$ mestres e $2,1 \%(n=1)$ doutor.

Mais da metade dos enfermeiros, especificamente $80,8 \%(n=38)$ estudaram sobre sepse durante a graduação. Destes, $39,47 \%(n=15)$ não lembravam o que estudaram. Em relação ao cuidado dispensado ao paciente em sepse, 
$40,4 \% \quad(n=19) \quad$ dos enfermeiros referiram ter dificuldades no cuidado como na identificação precoce baseado na suspeita clínica e $46,8 \%(n=22)$ afirmaram não se sentir capazes ou preparados para cuidar do paciente séptico.

Quando questionados

acerca da definição de sepse, 70,2\% $(\mathrm{n}=33)$ responderam erroneamente, não conseguiram descrever respostas compatíveis com a atual definição. Quanto à classificação da sepse, 70,2\% (n=33) participantes classificaram de outras formas, não sabiam ou não responderam (Tabela 1).

Tabela 1 - Conhecimento dos participantes acerca das classificações da sepse. Brasil, 2018.

Sepse e choque séptico

Não souberam

Sepse, sepse grave e Choque séptico

Outros (nomenclaturas não utilizadas para

classificação de sepse)

Não responderam

SIRS, sepse e/ou sepse grave e choque séptico

Sepse grave e choque Séptico

Total

$\begin{array}{cc}n & \% \\ 14 & 29,8 \\ 13 & 27,7 \\ 6 & 12,8 \\ 5 & 10,6 \\ 5 & 10,6 \\ 2 & 4,3 \\ 2 & 4,3 \\ 47 & 100\end{array}$

Quando questionados da Síndrome da Resposta

acerca dos sinais para o Inflamatória Sistêmica (SIRS) ou reconhecimento da sepse, 51\% $(n=24)$ ou elencaram sinais não ainda somente sinais de disfunção e não a combinação de sinais de SIRS utilizados para a identificação da e de disfunção orgânica (Tabela 2). sepse ou descreveram apenas sinais

Tabela 2 - Conhecimento dos participantes acerca dos sinais de sepse. Brasil, 2018.

\begin{tabular}{lcc}
\hline Conceitos descritos pelos entrevistados & $n$ & $\%$ \\
\hline Sinais de SIRS e Disfunção Orgânica & 23 & 48,9 \\
Sinais de SIRS e/ou disfunção orgânica e sinais não utilizados na & 15 & 31,9 \\
definição & & 10,6 \\
Sinais de SIRS somente & 5 & 8,5 \\
Sinais de disfunção orgânica somente & 4 & 100 \\
Total & 47 & \\
\hline
\end{tabular}

Quanto ao conhecimento da escala Quick-Sequential Organ Failure Assessment (Q-SOFA), utilizada para o manejo e avaliação do prognóstico do paciente séptico, $68,1 \%(n=32)$ disseram conhecer a 
mesma e desse total 21,8\% ( $\mathrm{n}=7) \quad$ (Tabela 3).

conheciam seus critérios de escore

Tabela 3 - Conhecimento dos participantes acerca da escala Q-SOFA e seus critérios de escore. Brasil, 2018.

\begin{tabular}{lcc}
\hline Conhecimento - escala Q-SOFA & $n$ & $\%$ \\
Sim & 32 & 68,1 \\
Não & 15 & 31,9 \\
Total & 47 & 100,0 \\
Conhecimento dos critérios de escore & 15 & 46,8 \\
Não responderam & 10 & 31,2 \\
Não souberam & 7 & 21,8 \\
Hipotensão, taquipneia e alteração do estado & 32 & 100 \\
mental & & \\
Total &
\end{tabular}

Em relação as ações antibioticoterapia na primeira hora preconizadas pelas recomendações após o reconhecimento, e 95,74\% no atendimento ao paciente com $(n=45)$ afirmaram que deve ser sepse, após o reconhecimento da feita coleta de sangue para mesma, 91,48\% ( $\mathrm{n}=43)$ afirmaram dosagem de lactato sérico (Tabela que deve ser feito 4).

Tabela 4 - Conhecimento dos participantes acerca das ações preconizadas para o tratamento da sepse. Brasil, 2018.

\begin{tabular}{lcc}
\hline Administração de antibiótico até a primeira hora & $n$ & $\%$ \\
\hline Sim & 43 & 91,5 \\
Não & 4 & 8,5 \\
Total & 47 & 100,0 \\
Coleta de sangue para dosagem de lactato sérico & & 63,8 \\
Sim & 30 & 36,2 \\
Não & 17 & 100,00 \\
Total & 47 & \\
\hline
\end{tabular}

0 perfil do enfermeiro de hospitais de ensino evidenciado em estudos nacionais é de profissionais do sexo feminino, com mais de um vínculo de trabalho e mais de 10 anos de tempo de formado e trabalho na instituição ${ }^{11-15}$, confirmando os resultados da presente investigação.

\section{Outros autores reafirmam} que enfermeiros que cursam pósgraduação têm um conhecimento adquirido aliado à prática, apresentando conhecimento satisfatório em pesquisas que se avalia conhecimento ${ }^{15,16}$. Entretanto, neste estudo o observado foi que, mesmo com este nível de formação entre a maioria 
dos participantes, os conhecimentos não foram satisfatórios e os profissionais ainda não se sentem mais qualificados para cuidar do paciente em sepse.

De acordo com a última atualização, a terceira definição pelo consenso internacional (Sepsis3) de 2016, a sepse pode ser classificada como sepse e choque séptico ${ }^{1}$. Porém, mais que a metade dos enfermeiros deste estudo desconhece essa nova definição e classificação. A ênfase encontrada nas respostas foi sobre a questão inflamatória e não na disfunção orgânica, mostrando conhecimento sobre identificação e classificação desatualizados, achado esse também constatado por outros autores ${ }^{8,11}$.

Somente os critérios de SIRS são muito abrangentes e inespecíficos, podem estar presentes em outras situações como pós-operatórios e quadros agudos, como pancreatite $\mathrm{e}$ traumas, além de não contemplarem a gravidade e complexidade da disfunção orgânica que ameaça a vida ${ }^{5}$. Sabese que a evolução de sepse para choque séptico está associada a uma mortalidade de mais de $40 \%$ e o fato de a diferença entre a sepse e o choque séptico não estar elucidada entre os profissionais pode comprometer 0 reconhecimento da disfunção orgânica e acarretar na perda de um momento oportuno de intervenção, uma janela clínica de tratamento, permitindo melhor evolução ${ }^{17}$.

De acordo com o protocolo gerenciado publicado pelo Instituto Latino Americano de Sepse para a confirmação de um caso é preciso de um foco infeccioso suspeito ou confirmado, pelo menos dois critérios de SIRS e/ou pelo menos um sinal de disfunção orgânica6 . Metade dos participantes não discriminou estes critérios em conjunto e há evidência em mais estudos $^{8,15}$ semelhantes sobre a dificuldade dentre os enfermeiros para identificação precoce das alterações sistêmicas causadas pela sepse. Dessa forma, estes profissionais não têm clareza da síndrome séptica muito menos do seu grau de complexidade de modo que essa deficiência compromete a detecção precoce, o tratamento correto e oportuno e 
consequentemente 0 prognóstico do paciente.

A disfunção orgânica recebe grande destaque no Sepsis-3 por ser o mecanismo chave da mortalidade na sepse. Dessa forma, este consenso preconiza a utilização de uma escala que avalia a disfunção sequencial dos órgãos, a Sequential Organ Failure Assessement (SOFA) em suas versões normal e curta (QUICK-SOFA/Q-SOFA) ${ }^{1}$. A escala QSOFA auxilia na rápida identificação e confirmação de um caso de sepse, é primordial no manejo e tratamento deste paciente logo, conhecê-la facilita o atendimento do doente séptico ${ }^{1}$.

Outros estudos 8,16 se preocuparam em avaliar o uso da Q-SOFA, porém não investigaram se os enfermeiros conhecem seus critérios. Entre os enfermeiros deste estudo, $68,1 \%$ conheciam a escala, mas desse total apenas $21,8 \%$ conheciam e apontaram seus critérios. Considerando a importância dessa avaliação, o desconhecimento é uma barreira, uma vez que, essa avaliação favorece a detecção precoce e consequentemente, a breve instituição de medidas terapêuticas ${ }^{17}$. Além disso, essa escala em sua versão curta avalia apenas a presença de hipotensão, taquipneia e alteração do estado mental, critérios de fácil verificação, não sendo necessário resultados de exames, como na sua versão normal, não retardando assim a identificação, o que confere grande praticidade $\mathrm{e}$ aplicabilidade ${ }^{4}$.

0 protocolo gerenciado de sepse também preconiza que no pacote de atendimento ao paciente séptico nas primeiras três horas deve-se administrar antibiótico, realizar reposição volêmica e colher sangue para dosagem de lactato sérico e outros exames ${ }^{6}$. Em quase totalidade das respostas, os participantes do presente estudo afirmaram a prática destas ações para o tratamento do paciente, demonstrando que essas medidas ocorrem, como também relatado por enfermeiros de acordo com a literatura em outras realidades de investigação ${ }^{16}$.

Mas é possível inclusive refletir que talvez esses enfermeiros apenas "fazem"15, instituem as medidas de tratamento, mas pouco entendem a 
fisiopatologia e a clínica apresentada pelo doente, não associam seu quadro clínico a exacerbada explosão respiratória e suas consequências e não exercem o pensamento e julgamento clínico e crítico acerca de todo o processo por ele assistido. Um fazer enfermagem sem pensar enfermagem.

O Conselho Regional de Enfermagem de São Paulo reconhece as dificuldades encontradas por enfermeiros nas instituições de saúde para implementar correta e sistematicamente os protocolos de otimização precoce guiados por metas para a sepse ${ }^{5}$. As respostas dos enfermeiros dessa instituição evidenciaram que eles conhecem o tema de maneira geral, porém indicam que, no contexto onde foi realizado o estudo, um hospital público de ensino, inexistem protocolos sistematizados e gerenciados, além da falta de treinamentos e desenvolvimentos de ações institucionais relacionados à sepse. Os hospitais escola são caracterizados por albergar campos de aulas práticas e estágios, para a formação de futuros profissionais, e por esse motivo, além dos aspectos éticos no cuidado à saúde, esperase encontrar profissionais capacitados, tanto em relação a sepse como nas demais demandas em saúde, com familiaridade e adesão à prática baseada em evidência, já que complicações dessa natureza não são rara e requer a intervenção do enfermeiro ${ }^{18}$.

$$
\text { A Campanha de }
$$

Sobrevivência da Sepse recomenda fortemente que as instituições de saúde possuam estratégias para a identificação precoce e programas de melhoria assim como treinamentos, capacitações e indicadores de qualidade no que diz respeito a sespse ${ }^{5}$.

Além disso, as habilidades e competências do enfermeiro devem acompanhar a importante responsabilidade de avaliar criteriosamente $\mathrm{o}$ paciente $\mathrm{e}$ participar do processo de tomada de decisão junto a equipe multiprofissional, com objetivo de garantir o trabalho em equipe em consonância com as recomendações atuais para o manejo da sepse ${ }^{19}$. Ademais, é de extrema importância que 0 enfermeiro não meça 
Conhecimento de enfermeiros sobre sepse...

esforços para que isso aconteça certo de que esse empenho trará melhores práticas relacionadas a bons prognósticos 20,21 .

Salienta-se que o estudo apresentou limitações considerando o pequeno número de participantes. Entretanto, a pesquisa trouxe importantes elementos para repensar a assistência nesse perfil de hospital.

\section{CONCLUSÃO}

Os participantes do estudo apresentam conhecimento insatisfatório acerca da definição de sepse e sua classificação, bem como sobre os sinais e sintomas para identificação da mesma. Para além dos aspectos semelhantes à literatura, este estudo apresenta aspectos da identificação precoce das alterações sistêmicas causadas pela sepse, não somente relacionadas ao profissional, mas no que tange as instituições de saúde, como a falta de protocolos e treinamentos.

É evidente a necessidade da implantação de protocolos que objetivem 0 atendimento otimizado, assertivo e individualizado, de forma a promover um cuidado seguro, embasado em evidências científicas, em diretrizes e recomendações atuais para 0 reconhecimento e tratamento precoce da sepse.

\section{REFERÊNCIAS}

1. Singer $M$, Deutschman CS, Seymour CW, Shankar-Hari $M$, Annane $D$, Bauer $M$, et al. The third international consensus defenitions for sepsis and septic shock (Sepsis-3). JAMA. 2016; 315(8):801-810.

2. Roth GA, Abate $\mathrm{D}$, Abate $\mathrm{KH}$, Abay SM, Abbafati C, Abbasi N, et al. Global, regional, and national age-sex-specific mortality for 282 causes of death in 195 countries and territories, 1980-2017: a systematic analysis for the Global Burden of Disease Study 2017. The Lancet. 2018; 392:1736-88.

3. DATASUS. Mortalidade por septicemia. Brasília: Ministério da Saúde; 2018.

4. Rhodes A, Evans LE, Alhazzani W, Levy MM, Antonelli M, Ferrer R, et al. Surviving sepsis campaign: 
international guidelines for management of sepsis and septic shock: 2016. Intensive Care Med. 2017; 43:304-377.

5. Conselho Regional de Enfermagem. Sepse um problema de saúde pública. Atuação e colaboração da enfermagem na rápida identificação e tratamento da doença. São Paulo - Brasil. [Internet] 2017 [cited 2018 Jun 6]. Available from: <https://www.ilas.org.br/assets /arquivos/ferramentas/livrosepse-um-problema-de-saudepublica-coren-ilas.pdf>.

6. Instituto Latino Americano de Sepse. Implementação de Protocolo Gerenciado de Sepse. Protocolo clínico Atendimento ao paciente adulto com sepse / choque séptico. [Internet] 2018 [cited 2018 nov 7]. Available from:

<https://ilas.org.br/assets/arqui vos/ferramentas/protocolo-detratamento.pdf $>$.

7. Instituto Latino Americano de Sepse (ILAS). Relatório Nacional. Protocolo Gerenciados de Sepse. Sepse Grave e Choque Séptico 2005-2015. São Paulo; 2016.
8. Goulart LS, Ferreira Júnior MA, Sarti ECFB, Sousa AFL, Ferreira AM, Frota OP. Os enfermeiros estão atualizados para o manejo adequado do paciente com sepse? Esc Anna Nery. 2019; 23(4):e20190013.

9. Pedroso KKA, Oliveira SA, Machado RC. Validação de protocolo assistencial ao paciente séptico na Unidade de Terapia Intensiva. Rev Bras Enferm. 2018; 71(3):1106-1114.

10. Pedro TCS, Morcillo AM, Baract ECE. Etiologia e fatores prognósticos da sepse em crianças e adolescentes admitidos em terapia intensiva. Rev Bras Ter Intensiva. 2015; 27(3):240-246.

11. Melech CS, Paganini MC. Avaliação do conhecimento de médicos e equipe de enfermagem nas ocorrências de sepse. Rev Med UFPR. 2016; 3(3):127-132.

12. Pedrosa KKA, Oliveira SA, Machado RC. Validation of a care protocol for the septic patient in the Intensive Care Unit. Rev Bras Enferm. 2018; 71(3):1106-14. 
Conhecimento de enfermeiros sobre sepse...

13. Leal LA, Soares MI, Silva BR, Chaves LDP, Camelo SHH. Desafios para desenvolver competências no âmbito hospitalar. REME Rev Min Enferm. 2018; 22:e-1099.

14. Cárnio EC. Novas Perspectivas no Tratamento do Paciente com Sepse. Rev latinoam enferm. 2019; 27:e3082.

15. Salviano MEM, Nascimento PDFS, Paula MA, Vieira CS, Frison SS, Maia MA, et al. Epistemology of nursing care: a reflection on its foundations. Rev Bras Enferm. 2016; 69(6):1172-7.

16. Kochhan SI, Mello AS, Dani C, Forgiarini Júnior LA. Adesão ao protocolo de sepse em um serviço de emergência relacionado à taxa de mortalidade intra-hospitalar. Rev Eletr Acervo Saúde. 2020; 38:e1856.

17. Cecconi $M$, Evans L, Levy $M$, Rhodes A. Sepsis and septic shock. Lancet. 2018; 392(10141):75-87.

18. Araújo KM, Leta J. Os hospitais universitários federais e suas missões institucionais no passado e no presente. Hist Ciênc Saúde. 2014: 21(4):12611281.

19. Cruz YV, Cardoso JDC, Cunha CRT, Vechia ADRD. Perfil da morbimortalidade da unidade de terapia intensiva de um hospital universitário. J Health NPEPS. 2019; 4(2):230-239.

20. Garrido F, Tieppo L, Pereira MDS, Freitas R, Freitas WM, Filipini $R$, et al. Ações do enfermeiro na identificação precoce de alterações sistêmicas causadas pela sepse grave. ABCS Health Sci. 2016; $42(1): 15-20$.

21. Lima JCC, Moraes-Filho IM, Santos TN, Silva CS, Melchior LMR, Sousa TV. Sepse e choque seqptico: compreensaro de enfermeiros de um hospital escola de grande porte. REVISA. 2020; 9(2):254-61. 
Conflito de interesses: Os autores declaram não haver conflito de interesses.

\section{Participação dos autores:}

- Concepção: Sousa TV, Melchior LMR.

- Desenvolvimento: Sousa TV, Melchior LMR, Bezerra MLR, Carvalho Filha FSS, Santos OP, Pereira MC, Félis KC, Moraes Filho IM.

- Redação e revisão: Sousa TV, Melchior LMR, Moraes Filho IM.

Como citar este artigo: Sousa TV, Melchior LMR, Bezerra MLR, Carvalho Filha FSS, Santos OP, Pereira MC, et al. Conhecimento de enfermeiros sobre sepse e choque séptico em um hospital escola. J Health NPEPS. 2020; 5(1):132-146.

Submissão: $18 / 03 / 2020$

Aceito: $23 / 05 / 2020$

Publicado: $01 / 06 / 2020$ 